\title{
Understanding Cosmological Measurements with a large number of mock galaxy catalogues.
}

\author{
M. Manera ${ }^{1} \dagger$, W. J. Percival ${ }^{2}$, Ashley Ross ${ }^{2}$, R. Tojeiro ${ }^{3}$,
} L. Samushia ${ }^{2}$, C. Howlett ${ }^{2}$ M. Vargas-Magaña ${ }^{4}$ and A. Burden ${ }^{2}$, for the SDSS-III BOSS Galaxy Working Group.

${ }^{1}$ Department of Physics \& Astronomy, University College London, Gower St, London WC1E 6BT, UK

${ }^{2}$ Institute of Cosmology and Gravitation, Dennis Sciama Building, University of Portsmouth, Burnaby Road, Portsmouth, PO1 3FX, UK

${ }^{3}$ School of Physics \& Astronomy, St Andrews University, North Haugh St Andrews, KY16 9SS, UK

${ }^{4}$ Department of Physics, Carnegie Mellon University, 5000 Forbes Avenue, Pittsburgh, PA 15213, USA

\begin{abstract}
Mock galaxy catalogues are essential to the error analysis of cosmological measurements from big galaxy surveys covering thousands of square degrees in the sky, like BOSS, WiggleZ, DES, or Euclid. The PTHalos mock galaxy catalogues were used in the BOSS survey to analyse the BAO measurement from the CMASS $(z \sim 0.57)$ and LOWZ $(z \sim 0.32)$ galaxy samples, which provided the best estimate to date of the cosmic distance scale from galaxy surveys at these redshifts. We review the PTHalos mocks galaxy catalogues and their key contributions to these analyses.
\end{abstract}

Keywords. cosmology: large scale structure of the universe, distance scale; methods: simulations, statistical

\section{The BAO scale measurement from BOSS galaxy samples}

The Baryon Acoustic Oscillation Survey (BOSS) is a spectroscopic survey that targets galaxies from SDSS-III (Eisenstein et al. 2011) imaging. BOSS targets two galaxy samples, CMASS $(z \sim 0.57)$ and LOWZ $(z \sim 0.32)$, which respectively cover 8,976 and 7, 341 square degrees in the Data Release 11 (DR11).

BOSS has detected and accurately measured the Baryon Acoustic Oscillations (BAO) in the two-point correlation functions and the power spectra of both the CMASS and LOWZ DR11 galaxy samples (Anderson et al. 2014). The BAO scale is given by the distance the sound waves travel before the coupling between baryons and radiation breaks down, $r_{d}$, which is $103.8 \mathrm{~h}^{-1} \mathrm{Mpc}$ in Anderson et al. (2014) fiducial flat LCDM cosmology.

The cosmic distance measurement, $D_{V}$ is related to the observed BAO scale, and is a combination of the angular distance, $D_{A}$, and the Hubble parameter $H(z)$,

$$
D_{V}=\left[c z(1+z)^{2} D_{A}^{2}(z) H^{-1}(z)\right]^{1 / 3} .
$$

The corresponding cosmic distance measurement for the CMASS galaxy sample is given by $D_{V} r_{d, f i d}=\alpha D_{V, f i d} r_{d}=(2056 \pm 20) r_{d}$ (which is at $1 \%$ accuracy); and for the LOWZ galaxy sample by $D_{V} r_{d, f i d}=(1264 \pm 25) r_{d}$ (which is sub $2 \%$ accuracy), where $\alpha$ parametrizes the position of the BAO peak.

\section{$\dagger$ email:m.miret@ucl.ac.uk}



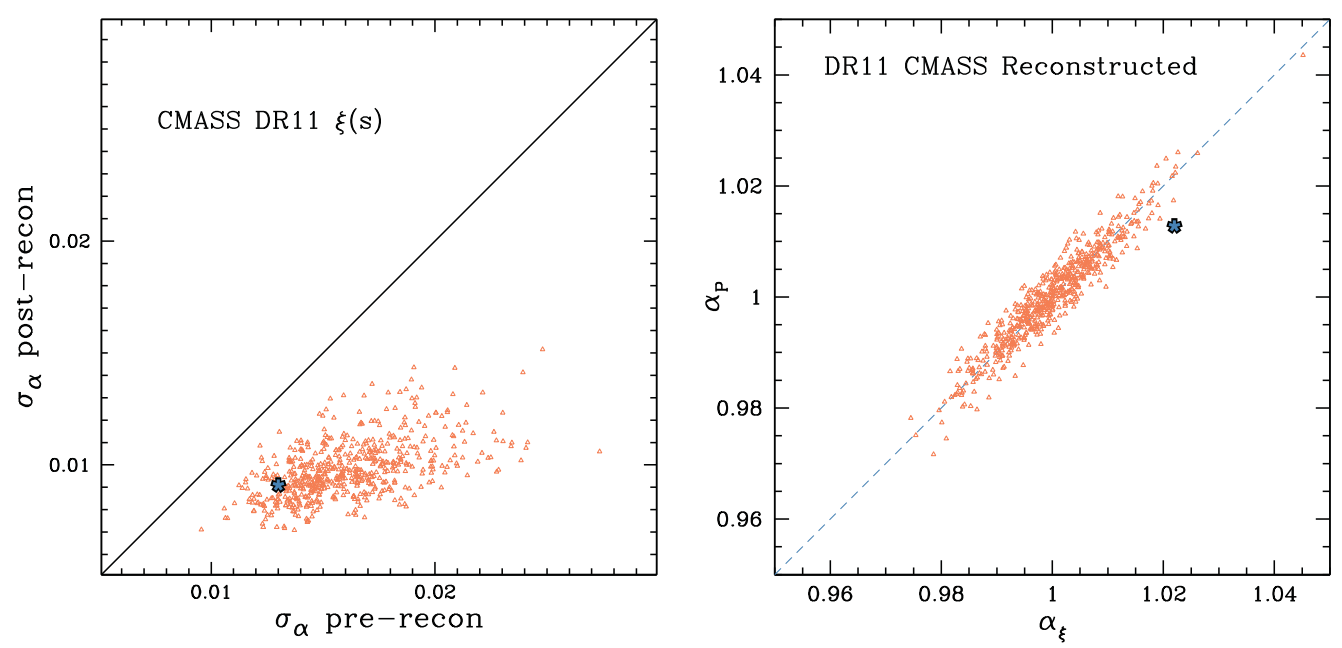

Figure 1. Left. Comparison between the errors of the BAO peak, $\alpha$, before and after applying reconstruction. Right. Comparison between the BAO peak position $\alpha$ estimated from the correlation function $\xi(s)$ and that estimated from the power spectrum $P(k)$. Both panels show results from the 600 CMASS PThalos mock catalogues. The results from data are shown as stars. For more details see Anderson et al. 2014.

\section{The creation of PTHalos mock galaxy catalogues}

The 600 (CMASS) and 1,000 (LOWZ) PTHalos mock galaxy catalogues used to analyse the clustering of BOSS galaxy samples have been constructed in four main steps (Manera et al. 2013 \& 2014):

- First, dark matter particle fields are created using 2nd order Lagrangian Perturbation Theory (2LPT). This step is what makes this method fast.

- Second, using a Friends of Friends (FoF) algorithm (with the appropriate linking length from theory) halos are found.

- Third, these halos are then populated with galaxies using a Halo Occupation Distribution prescription that fits the data

- Fourth, finally the observational masks are applied.

\section{PTHalos mocks and the BAO measurement analysis}

The reconstruction technique (see eg Padmanabhan et al. 2012) moves galaxies back in time in order to recover a more linear correlation function, and thus a more prominent BAO peak, i.e, one less smoothed by the non-linear evolution. The PThalos mock catalogues have been used to characterize the gain in accuracy when using this technique (Anderson et al. 2014). The results for the CMASS sample are shown in the left panel of Fig 1; points show the mocks, and the star shows the data.

The BAO scale can be measured using different estimators. The PTHalos mocks have been used to characterize the correlation between them (Anderson et al.2014), enabling their combination. This is shown in the right panel of Fig 1. Mock galaxy catalogues have also been essential to understand systematics (Ross et al. 2012, Vargas-Magaña et al. 2014) and to choose the best binning for the BAO measurement (Percival et al. 2014). 


\section{Finite number of mock catalogues}

Having a large number of mock galaxy catalogues allows for the computation of covariance matrices. However, as the number of mock catalogues is finite, an accurate estimation of the cosmic distance measurement error requires correcting for the error in the inverse covariance matrix caused by the finite number of mocks available (Taylor et al.2012), and for the derived parameter error. Given a covariance matrix, $C$, which has been computed with $n_{s}$ mock catalogues for correlation function or power spectrum with $n_{b}$ bins, the unbiased estimator of the inverse covariance $\Psi$ is given by:

$$
\Psi=\frac{n_{s}-n_{b}-2}{n_{s}-1} C^{-1}
$$

Furthermore, the error corrections for the derived parameters depend on the way in which the parameter error is determined, and are different if this is calculated from the distribution of recovered values from the mocks, or the likelihood surface (Percival et al. 2014). The correction factor to the estimate of the variance of a parameter given by the standard method of integrating over the likelihood

$$
c_{1}=\frac{1+B\left(n_{s}-n_{p}\right)}{1+A+B\left(n_{p}+1\right)}
$$

where $A=\left[0.5\left(n_{s}-n_{b}-1\right)\left(n_{s}-n_{b}-4\right)\right]^{-1}$ and $B=0.5 A\left(n_{s}-n_{b}-2\right)$ If the variance is computed from the distribution of the best fit values of the mocks catalogues that have also been used to compute the covariance matrix, then the correction of the variance requires an extra factor (Percival et al. 2014) of $c_{2}=\frac{n_{s}-1}{n_{s}-n_{b}-2}$.

\section{Acknowledgements}

SDSS-III is managed by the Astrophysical Research Consortium for the Participating Institutions of the SDSS-III Collaboration including the University of Arizona, the Brazilian Participation Group, Brookhaven Na- tional Laboratory, University of Cambridge, Carnegie Mellon University, University of Florida, the French Participation Group, the German Participation Group, Harvard University, the Instituto de Astrofisica de Canarias, the Michigan State/Notre Dame/JINA Participation Group, Johns Hopkins University, Lawrence Berkeley National Laboratory, Max Planck Institute for Astrophysics, Max Planck Institute for Extraterrestrial Physics, New Mexico State University, New York University, Ohio State University, Pennsylvania State University, University of Portsmouth, Princeton University, the Spanish Participation Group, University of Tokyo, University of Utah, Vanderbilt University, University of Virginia, University of Washington, and Yale University.

\section{References}

Anderson, et al. 2014, MNRAS 441, 24 BOSS collaboration paper

Eisenstein, D. J., Weinberg, D. H., Agol, E., et al. 2011, AJ, 142, 72

Manera, et al. 2013 MNRAS 428, 1036

Manera, et al. 2014, arXiv 1401.4171

Padmanabhan, et al. 2012, MNRAS,427,2132

Percival, et al. 2014, MNRAS 439,2531

Ross, et al. 2012 MNRAS,424,564

Taylor, et al. 2012, MNRAS,432,1928

Vargas-Magana, et al. 2014, arXiv:1312.4996 